\title{
Strangeness in Lattice QCD
}

\author{
Hartmut Wittig $\ddagger$ \\ CERN, Theory Division, CH-1211 Geneva 23, Switzerland
}

\begin{abstract}
The status of lattice calculations in the light hadron sector is reviewed. Special emphasis is given to recent lattice determinations of the mass of the strange quark. The impact of non-perturbative renormalization and control over lattice artefacts on the attainable precision is discussed in detail. Furthermore the influence of dynamical quark effects is assessed.
\end{abstract}

\section{Introduction}

Quantum Chromodynamics (QCD) is widely accepted as the theory describing the strong interaction. It is formulated in terms of quarks and gluons and contains relatively few free parameters, namely the gauge coupling $g$ and the masses $m_{\mathrm{u}}, m_{\mathrm{d}}, m_{\mathrm{s}}, \ldots$ of the various quarks. These parameters must be fixed by using experimental input: a typical example is the determination of the sum of quark masses $\left(m_{\mathrm{u}}+m_{\mathrm{s}}\right)$, using the experimental value of the kaon mass, $m_{\mathrm{K}^{+}}$:

$$
m_{\mathrm{K}^{+}} \mapsto\left(m_{\mathrm{u}}+m_{\mathrm{s}}\right) .
$$

Clearly, in order to connect the two sides of this relation one has to "solve QCD" for the case at hand.

The QCD coupling constant $g$, which describes the coupling strength, depends on the momentum transfer $q$ between quarks and gluons. The well-known property of "asymptotic freedom" implies that $g$ decreases for large $q$. By contrast, the coupling becomes large when momentum transfers of the order of typical hadronic scales are considered, say, $q \approx 1 \mathrm{GeV} / c$. As a consequence, perturbation theory in $g^{2}$ becomes an inadequate tool to study QCD at low energies. Hence, a non-perturbative treatment is required in order to understand hadronic properties on a quantitative level. The formulation of QCD on a (Euclidean) space-time lattice [1] provides such a non-perturbative method to compute the relations between experimentally accessible quantities and the parameters of QCD through numerical simulations.

Here I restrict myself to QCD at zero temperature, focusing on the spectrum of light hadrons and the determination of the strange quark mass from first principles. A more general overview of the status of lattice calculations can be obtained from the

$\ddagger$ On leave of absence from: Theoretical Physics, University of Oxford, 1 Keble Road, Oxford OX1 3NP, UK

Invited talk presented at V International Conference on Strangeness in Quark Matter, "Strangeness 2000", Berkeley, CA, 20-25 July 2000 
proceedings of the annual Conference on Lattice Field Theory [2] and other review talks $[3-6]$.

In the next section I shall outline the basic concepts of the lattice formulation. Section 3 contains results from recent benchmark calculations of the light hadron spectrum. In section 4 recent determinations of the strange quark mass are described. Finally, section 5 contains some concluding remarks.

\section{Basic concepts}

The lattice formulation of QCD replaces the familiar continuous Minkowski space-time by a discretized, Euclidean version with finite volume $L^{3} \cdot T$. Points in space-time are separated by a finite distance $a$, the lattice spacing. The inverse lattice spacing, $a^{-1}$, acts as an UV cutoff, which regularizes the infinities that are typically encountered in Quantum Field Theory. The quark and antiquark fields $\psi(x), \bar{\psi}(x)$ are associated with the sites of the lattice, whereas the gauge field is represented by the so-called link variable $U_{\mu}(x)$, which connects neighbouring lattice sites, and is an element of the gauge group $\mathrm{SU}(3)$. After choosing a suitable, gauge-invariant discretization of the QCD action

$$
S[U, \bar{\psi}, \psi]=S_{\mathrm{G}}[U]+S_{\mathrm{F}}[U, \bar{\psi}, \psi],
$$

where $S_{\mathrm{G}}$ is the lattice action of pure Yang-Mills theory and $S_{\mathrm{F}}$ denotes the lattice fermion action, one may define the expectation value of an observable $\Omega$ as

$$
\langle\Omega\rangle=\frac{1}{Z} \int D[U] D[\bar{\psi}] D[\psi] \Omega \exp \left(-S_{\mathrm{G}}-S_{\mathrm{F}}\right) .
$$

Here the normalization of the functional integral $Z$ is determined by requiring $\langle 1\rangle=1$. After performing the integration over fermionic fields the expression for the expectation value becomes

$$
\langle\Omega\rangle=\frac{1}{Z} \int \prod_{x, \mu} d U_{\mu}(x) \prod_{\mathrm{f}} \operatorname{det}\left(D+m_{\mathrm{f}}\right) \Omega \exp \left(-S_{\mathrm{G}}\right),
$$

where $D$ is the lattice Dirac operator and $m_{\mathrm{f}}$ is the mass of quark flavour $f$. Thus, the discretization procedure has given a meaning to the functional integration over gauge fields, which reduces to an ordinary multiple-dimensional integral over group elements. The various steps leading to eq. (4) can be treated in a gauge-invariant manner, and hence the lattice formulation represents a regularization procedure that preserves the gauge invariance of QCD. Furthermore, the definition of physical observables does not rely on perturbation theory. Therefore eq. (4) forms the basis for a non-perturbative, stochastic evaluation of expectation values of physical observables using numerical simulations.

Despite an enormous increase in computer power, there remain several major difficulties that make realistic simulations of QCD a hard task. Perhaps the biggest challenge is the inclusion of dynamical quark effects: the evaluation of the fermionic determinant in eq. (4) in numerical simulations is still very costly, even on today's massively parallel computers. In early simulations of lattice QCD the determinant was therefore set equal to 1 , a choice whose physical interpretation corresponds to neglecting quark loops in the evaluation of $\langle\Omega\rangle$. Although this represents a rather drastic assumption about the influence of quark-induced quantum effects, the quenched approximation works surprisingly well, as we shall see later. However, it is clearly desirable to develop simulation algorithms that allow for a 
more efficient evaluation of the fermionic determinant. With present algorithms, the cost of simulating "full" QCD is roughly a thousand times higher than that of the corresponding quenched simulation.

An indirect consequence of using the quenched approximation is the so-called scale ambiguity. That is, the calibration of the lattice spacing in physical units, $a^{-1}[\mathrm{MeV}]$, depends on the quantity $Q$, which is used to set the scale

$$
a^{-1}[\mathrm{MeV}]=\frac{Q[\mathrm{MeV}]}{(a Q)}, \quad Q=f_{\pi}, m_{N}, m_{\rho}, \ldots
$$

This ambiguity arises because different quantities $Q$ are affected by quark loops in different ways. Clearly, dimensionful quantities such as particle masses are directly affected by the scale ambiguity.

Another problem one has to address are lattice artefacts (cutoff effects). Let $\Omega$ denote the mass of a hadron in units of, say, the nucleon mass. Then the expectation values on the lattice and in the continuum differ by corrections of order $a^{p}$ :

$$
\langle\Omega\rangle^{\text {lat }}=\langle\Omega\rangle^{\text {cont }}+O\left(a^{p}\right),
$$

where the value of integer $p$ in the correction term depends on the chosen discretization of the QCD action. Values of $a$ that can currently be simulated lie in the range $a \approx 0.2-0.05 \mathrm{fm}$. The size of the correction term can in some cases be as large as $20 \%$, depending on the quantity and the chosen discretization. An extrapolation to the continuum limit, $a \rightarrow 0$, is then required to obtain the desired result. Surely this extrapolation is much better controlled if the discretization avoids small values of $p$.

Finally there are restrictions on the quark masses $m_{\mathrm{f}}$ that can be simulated. In general the following inequalities should be satisfied in any simulation:

$$
a \ll \xi \ll L,
$$

where $L$ is the spatial extent of the lattice volume. The quantity $\xi$ denotes the correlation length of a typical hadronic state and serves as a measure of the quark mass. The inequality on the right places restrictions on the light quark masses that can be simulated: if those are too light one may suffer from finite-size effects, since $\xi$ becomes large. Typical spatial extensions of $L \approx 1.5-3 \mathrm{fm}$ imply that the physical pion mass cannot be reached. The left inequality restricts the masses of heavy quarks. Since $a^{-1} \approx 2-4 \mathrm{GeV}$, it is clear that relativistic $b$-quarks cannot be simulated. One therefore relies on extrapolations in $m_{\mathrm{f}}$ to connect to the physical $u, d$ and $b$ quarks.

\section{Hadron spectroscopy}

In many ways the calculation of the mass spectrum of light hadrons is a benchmark of lattice QCD. A comparison of the experimentally observed spectrum with the results from an "ideal" lattice simulation of QCD, in which dynamical quark effects, lattice artefacts and quark mass dependences are all sufficiently controlled, would represent a stringent test of QCD as the underlying theory of the strong interaction. Alternatively, such a comparison enables us to assess the inherent systematics of current simulations, notably the effects of dynamical quarks. In particular one may investigate the quality of the quenched approximation. 


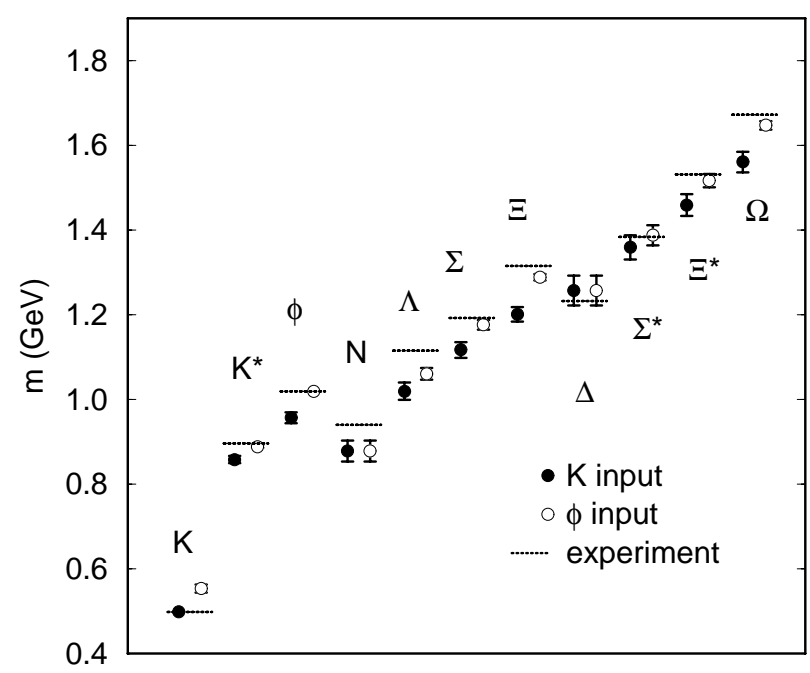

Figure 1. The quenched hadron spectrum from ref. [7] compared with experiment (dashed lines).

\subsection{Quenched light hadron spectrum}

Recently the CP-PACS Collaboration presented a precision calculation of the light hadron spectrum in quenched QCD [7], which superseded an earlier study by GF11 [8]. The findings of CP-PACS are summarized in the plot shown in Fig. 1. The main features of the spectrum are well reproduced by the quenched lattice data. Bearing in mind that only two input quantities have been used, namely the mass of the $\rho$-meson to set the scale and the mass of a strange meson (either $m_{\mathrm{K}}$ or $m_{\phi}$ ) to fix the mass of the strange quark, the fact that the masses of so many hadrons are predicted quite accurately represents a major achievement in the understanding of QCD. Nevertheless, one finds small but significant deviations from the experimentally observed spectrum. For instance, the ratio of the nucleon and $\rho$ masses is calculated as

$$
m_{\mathrm{N}} / m_{\rho}=1.143 \pm 0.033,
$$

which is $6.7 \%(2.5 \sigma)$ below the experimental value of 1.218 . Similarly, vectorpseudoscalar mass splittings such as $m_{\mathrm{K}^{*}}-m_{\mathrm{K}}$ are too small by $10-16 \%(4-6 \sigma)$, depending on whether $m_{\mathrm{K}}$ or $m_{\phi}$ is used to fix the strange quark mass. This implies that, for the first time, a significant deviation between the quenched QCD spectrum and nature is detected. Thus the conclusion is that quenched QCD describes the light hadron spectrum at the level of $10 \%$. However, it also shows that the quenched approximation works surprisingly well, since the discrepancy is fairly mild. This has important consequences for lattice predictions of some phenomenologically interesting quantities, for which one has to rely on the quenched approximation for some time.

Although the CP-PACS results represent a real benchmark in terms of statistics, parameter values and lattice volumes, further corroboration of these findings is required. Recent calculations employing different discretizations have largely confirmed the findings of [7]: the MILC Collaboration [9] has used staggered fermions [10] and finds a value for $m_{\mathrm{N}} / m_{\rho}$ in the continuum limit of

$$
m_{\mathrm{N}} / m_{\rho}=1.254 \pm 0.018 \text { (stat) } \pm 0.028 \text { (syst). }
$$


This is in broad agreement with experiment, but the difference to the CP-PACS result amounts to only $2 \sigma$. A recent calculation by UKQCD using $O(a)$ improved Wilson fermions [11] finds $m_{\mathrm{N}} / m_{\rho}=1.26_{-14}^{+8}$. Whereas the error is too large to detect a significant deviation, UKQCD's results for the spectrum also indicate that the quenched light hadron spectrum agrees with experiment at the level of $10 \%$.

\subsection{Beyond the quenched approximation}

An obvious question is whether sea quark effects can account for the observed deviation of the quenched light hadron spectrum from experiment. Recently several collaborations have studied the light hadron spectrum using $N_{\mathrm{f}}=2$ flavours of dynamical quarks, which are identified with the physical up and down quarks [12-16]. However, the masses of the dynamical quarks that can be simulated are quite large. A measure for the difference between the light quark masses found in nature and those used in dynamical simulations is provided by the pseudoscalar-to-vector mass ratio, $m_{\mathrm{PS}} / m_{\mathrm{V}}$. Current simulations are typically performed for $m_{\mathrm{PS}} / m_{\mathrm{V}}=0.6-$ 0.8 , whereas the physical value is $m_{\mathrm{PS}} / m_{\mathrm{V}}=m_{\pi} / m_{\rho}=0.169$. Therefore one relies on extrapolations in the sea quark mass to make contact with the physical case. In addition, it has not been possible to simulate a third dynamical quark with $m_{\mathrm{f}} \approx m_{\mathrm{s}}$ efficiently using known algorithms. Therefore, in most dynamical calculations an unphysical number of sea quarks has been used.

Despite these shortcomings one can perform systematic studies of the influence of dynamical quarks on the light hadron spectrum. CP-PACS [15] have studied the continuum limit of hadron masses computed for $N_{\mathrm{f}}=2$ and compared it with the results of the quenched light hadron spectrum discussed before. They find that the discrepancy with experiment is reduced from $6.5 \%$ to $1.4 \%$ for $m_{\phi}$ computed with $N_{\mathrm{f}}=2$. For $m_{\mathrm{K}^{*}}$ the gap decreases from $4.4 \%$ to $1.0 \%$. This demonstrates clearly that the experimentally observed spectrum is reproduced more closely when sea quarks are "switched on". Furthermore, results for the vector-pseudoscalar mass splitting reported by UKQCD [13] also show that lattice data for this quantity approach the experimental value as the sea quark mass is decreased.

Of course, the small remaining differences between QCD with $N_{\mathrm{f}}=2$ and experiment have to be explained. It is reasonable to assume that the unphysical value of $N_{\mathrm{f}}$ will have some influence. Further studies are also required to decide whether there is yet sufficient control over the extrapolations in the quark masses and those to the continuum limit.

\section{The mass of the strange quark}

Quark masses are important input parameters in many theoretical applications but, despite a great deal of activity, relatively little is known about their absolute values [17]. Here I shall describe calculations of current quark masses defined through the PCAC relation. For charged kaons it can be written as

$$
f_{\mathrm{K}} m_{\mathrm{K}}^{2}=\left(\bar{m}_{\mathrm{u}}+\bar{m}_{\mathrm{s}}\right)\left\langle 0\left|\bar{u} \gamma_{5} s\right| \mathrm{K}\right\rangle
$$

where the bars above the quark masses indicate that the "running" masses are considered, which depend on the energy scale. In order to determine the sum of up and strange quark masses using the experimental result for $f_{\mathrm{K}} m_{\mathrm{K}}^{2}$ one simply has to compute the matrix element $\left\langle 0\left|\bar{u} \gamma_{5} s\right| \mathrm{K}\right\rangle$ in a lattice simulation. However, in order 


\begin{tabular}{|c|c|c|c|c|}
\hline $\begin{array}{l}\text { Lattice } \\
\text { scheme: } m_{\text {lat }}(a)\end{array}$ & $\underset{a^{-1} \gg \mu_{0}}{\stackrel{\text { non-perturb. }}{\longrightarrow}}$ & $\begin{array}{l}\text { Intermediate } \\
\text { scheme: } \bar{m}_{\mathrm{X}}(\mu) \\
\mu_{0} \leq \mu \leq \bar{\mu}\end{array}$ & $\underset{\mu_{0}, a^{-1} \ll \bar{\mu}}{\text { perturbative }}$ & $\begin{array}{l}\overline{\mathrm{MS}} \text { scheme: } \\
\bar{m}_{\overline{\mathrm{MS}}}(\bar{\mu})\end{array}$ \\
\hline
\end{tabular}

Figure 2. Schematic relation between quark masses in lattice regularization and the $\overline{\mathrm{MS}}$ scheme through an intermediate renormalization scheme X.

to represent meaningful theoretical input, quark masses have to be renormalized, and hence their values depend on the adopted renormalization procedure. By convention quark masses are quoted in the $\overline{\mathrm{MS}}$ scheme of dimensional regularization at a reference scale, typically $\mu=2 \mathrm{GeV}$. This implies that the renormalized matrix element in the $\overline{\mathrm{MS}}$ scheme has to be determined; it is related to its lattice counterpart by

$$
\left\langle 0\left|\bar{u} \gamma_{5} s(\bar{\mu})\right| \mathrm{K}\right\rangle_{\overline{\mathrm{MS}}}=Z_{\mathrm{P}}^{\overline{\mathrm{MS}}}\left(g_{0}, a \bar{\mu}\right)\left\langle 0\left|\bar{u} \gamma_{5} s\right| \mathrm{K}\right\rangle_{\text {lat }} .
$$

Here $g_{0}$ is the bare coupling, and $\bar{\mu}$ is the subtraction point in the $\overline{\mathrm{MS}}$ scheme. The renormalization factor $Z_{\mathrm{P}}^{\overline{\mathrm{MS}}}$ is known to one-loop order in lattice perturbation theory. However, it is well known that lattice perturbation theory converges slowly, and in order to remove all doubts about the reliability of the matching procedure it is evident that a non-perturbative determination of the renormalization factor is required.

However, a non-perturbative normalization condition relating the matrix elements in lattice regularization and the $\overline{\mathrm{MS}}$ scheme cannot be formulated, since the latter is only defined to any given order of perturbation theory. This technical difficulty can be overcome by introducing an intermediate scheme $\mathrm{X}$ and considering a two-step matching procedure, as shown schematically in Fig. 2. The first part is the matching of the bare current quark mass $m_{\text {lat }}(a)$ to the running mass in the intermediate scheme $\bar{m}_{\mathrm{X}}(\mu)$. This amounts to computing $Z_{\mathrm{P}}^{\mathrm{X}}\left(g_{0}, a \mu_{0}\right)$ between the lattice scheme and scheme $\mathrm{X}$ at a fixed scale $\mu_{0}$. The second part is the determination of the scale dependence of the running mass $\bar{m}_{\mathrm{X}}(\mu)$ from $\mu_{0}$ up to very high energies, where the perturbative relation between $\bar{m}_{\mathrm{X}}$ and $\bar{m}_{\overline{\mathrm{MS}}}$ is expected to be reliable. Through this two-step process the use of lattice perturbation theory is completely avoided.

Two examples for such intermediate schemes have been proposed. The so-called "regularization-independent" (RI) scheme is described in [18]. Another intermediate scheme is defined using the Schrödinger Functional (SF) of QCD [19-21]. This scheme allows one to compute the scale dependence non-perturbatively over several orders of magnitude, using a recursive finite-size scaling technique. Once the scale dependence of the running mass in the SF scheme, $\bar{m}_{\mathrm{SF}}$, is known up to $\mu \approx 100 \mathrm{GeV}$ one can continue the scale evolution to infinite energy using the perturbative renormalization group functions and thereby extract the renormalization group invariant (RGI) quark mass $M$. At this point the matching to the $\overline{\mathrm{MS}}$ scheme is trivial, since $M$ is schemeindependent, i.e. it coincides in the SF and $\overline{\mathrm{MS}}$ schemes.

Figure 3 shows the non-perturbatively determined scale dependence of $\bar{m}_{\mathrm{SF}} / M$ computed in quenched QCD [22] and compares it to the perturbative scale evolution. The left-most data point in Fig. 3 corresponds to a scale $\mu_{0}=275 \mathrm{MeV}$. It is here that the matching between lattice regularization and the $\overline{\mathrm{MS}}$ scheme via the $\mathrm{SF}$ is completed, through a non-perturbative calculation of $Z_{\mathrm{P}}^{\mathrm{SF}}\left(g_{0}, a \mu_{0}\right)$ at fixed $\mu_{0}=275 \mathrm{MeV}$. All dependence on the intermediate SF scheme and the scale $\mu_{0}$ 


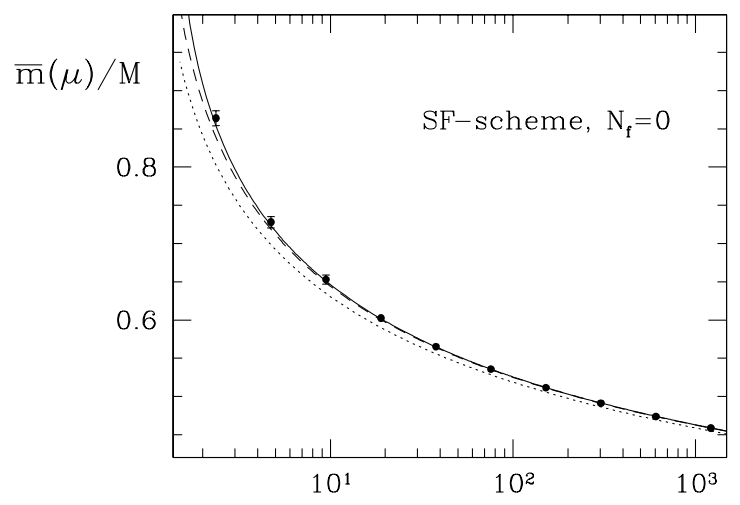

Figure 3. Non-perturbative scale evolution of $\frac{\mu}{m_{\mathrm{SF}}} / \Lambda_{M}$ computed in lattice simulations of the SF (solid circles). The lines correspond to the scale evolution computing using various orders of perturbation theory.

drops out in the total renormalization factor

$$
\frac{\bar{m}_{\mathrm{SF}}\left(\mu_{0}\right)}{M} \times Z_{\mathrm{P}}^{\mathrm{SF}}\left(g_{0}, a \mu_{0}\right)
$$

where $\bar{m}_{\mathrm{SF}}\left(\mu_{0}\right) / M=0.864 \pm 0.011$, as can be read off the left-most point in Fig. 3 [22]. The total renormalization factor is thus known with a precision of $1.5 \%$.

\subsection{The strange quark mass in quenched $Q C D$}

I shall now discuss the application of non-perturbative quark mass renormalization to compute the mass of the strange quark in the quenched approximation. Before discussing the details, it is useful to recall that mass ratios of light quarks are predicted quite accurately by Chiral Perturbation Theory (ChPT), for instance [23]

$$
\frac{M_{\mathrm{s}}}{\widehat{M}}=24.4 \pm 1.4, \quad \widehat{M}=\frac{1}{2}\left(M_{\mathrm{u}}+M_{\mathrm{d}}\right) .
$$

In order to determine $M_{\mathrm{s}}$, it is then sufficient to compute the sum $\left(M_{\mathrm{s}}+\widehat{M}\right)$ on the lattice and combine it with eq. (13).

Here I shall concentrate on the calculation performed by the ALPHA/UKQCD Collaboration [24]. By combining the PCAC relation, eq. (10), with the total renormalization factor, one obtains the sum of RGI quark masses $\left(M_{\mathrm{s}}+\widehat{M}\right)$ in units of the kaon decay constant $\S$

$$
\frac{M_{\mathrm{s}}+\widehat{M}}{f_{\mathrm{K}}}=\left(\frac{M}{\bar{m}_{\mathrm{SF}}} \cdot \frac{1}{Z_{\mathrm{P}}}\right) \times \frac{m_{\mathrm{K}}^{2}}{\left\langle 0\left|\bar{\ell} \gamma_{5} s\right| \mathrm{K}\right\rangle_{\text {lat }}}+O\left(a^{2}\right) .
$$

The dependence on the lattice spacing can be eliminated by performing a continuum extrapolation as shown in Fig. 4. Using the experimental value $f_{\mathrm{K}}=160 \pm 2 \mathrm{MeV}[17]$ one obtains the result for the sum of RGI quark masses in the continuum limit:

$$
M_{\mathrm{s}}+\widehat{M}=140 \pm 5 \mathrm{MeV} \text {. }
$$

This result can now be converted into $\bar{m}_{\mathrm{s}}^{\overline{\mathrm{MS}}}(\bar{\mu})$ at $\bar{\mu}=2 \mathrm{GeV}$. First, eq. (15) is combined with the prediction from ChPT, eq. (13). Then, the relation between the

$\S$ Here, $\ell$ denotes the Dirac spinor for the isospin-symmetric light quark. 


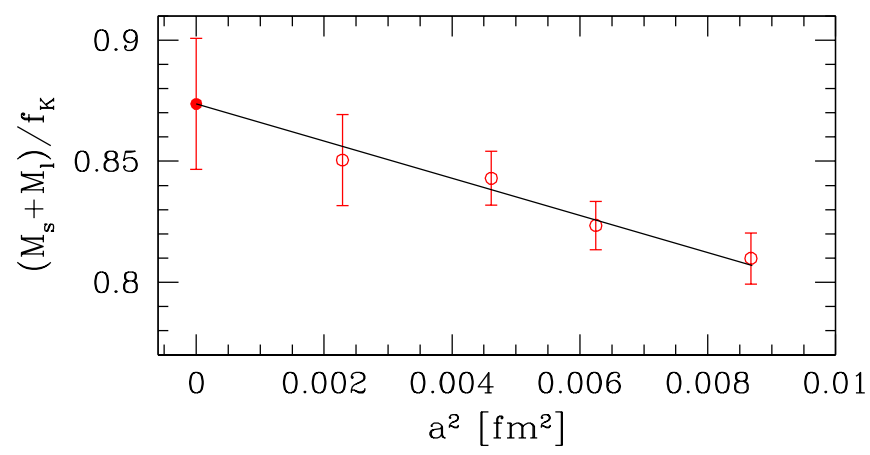

Figure 4. Continuum extrapolation of $\left(M_{\mathrm{S}}+\widehat{M}\right) / f_{\mathrm{K}}$ from ref. [24].

Table 1. Estimates for the quark masses $m_{\mathrm{S}}$ and $\widehat{m}$ in the $\overline{\mathrm{MS}}$ scheme in quenched $\mathrm{QCD}$. The choice of intermediate renormalization scheme $\mathrm{X}$ is also shown, where applicable.

\begin{tabular}{|c|c|c|c|c|}
\hline Collaboration & $\widehat{m}(2 \mathrm{GeV})$ & $m_{\mathrm{s}}(2 \mathrm{GeV})$ & $\mathrm{X}$ & $a \rightarrow 0$ \\
\hline CP-PACS [25] & $4.4(2)$ & $110(4)$ & & yes \\
\hline Becirevic et al [26] & $4.8(5)$ & $111(9)$ & RI & no \\
\hline Göckeler et al [27] & $4.4(2)$ & $105(4)$ & $\mathrm{SF}$ & yes \\
\hline Wingate et al [28] & & $130(11)(18)$ & $\mathrm{RI}$ & no \\
\hline ALPHA/UKQCD [24] & & $97(4)$ & $\mathrm{SF}$ & yes \\
\hline Blum et al [29] & & $96(26)$ & & yes \\
\hline JLQCD [30] & $4.23(29)$ & $106(7)$ & RI & yes \\
\hline Becirevic et al [31] & $4.5(4)$ & $111(12)$ & $\mathrm{RI}$ & no \\
\hline Giménez et al [32] & $5.7(1)(8)$ & $130(2)(18)$ & $\mathrm{RI}$ & no \\
\hline
\end{tabular}

running mass in the $\overline{\mathrm{MS}}$ scheme and the RGI quark mass is computed in 4-loop perturbation theory as

$$
\bar{m}^{\overline{\mathrm{MS}}}(\bar{\mu}) / M=0.7208 \text { at } \bar{\mu}=2 \mathrm{GeV} .
$$

This procedure yields the final result in the quenched approximation [24]

$$
\bar{m}_{\mathrm{s}}^{\overline{\mathrm{MS}}}(2 \mathrm{GeV})=97 \pm 4 \mathrm{MeV} \text {. }
$$

The quoted uncertainty of $\pm 4 \mathrm{MeV}$ contains all errors, except those due to quenching. The high precision of this result is a direct consequence of recent progress in lattice calculations, in particular the implementation of non-perturbative renormalization and control over lattice artefacts. As mentioned in section 2, the conversion into physical units is ambiguous in the quenched approximation. For $\bar{m}_{\mathrm{s}}^{\overline{\mathrm{MS}}}(2 \mathrm{GeV})$ the resulting uncertainty was estimated in ref. [24] to amount to $\sim 10 \%$.

Table 1 contains a compilation of recent results for $m_{\mathrm{s}}$ and $\widehat{m}$ obtained in the quenched approximation. Direct comparisons of these results should be made with care, since systematic errors have not been estimated in a uniform manner. Also, the conversion into physical units has been performed using different quantities. This manifests itself in the typical spread of the central values, which is of the order of $10 \%$, consistent with the above estimate. It is still quite remarkable that lattice estimates for light quark masses have stabilized, which represents a big improvement with respect to the situation before 1998 . 


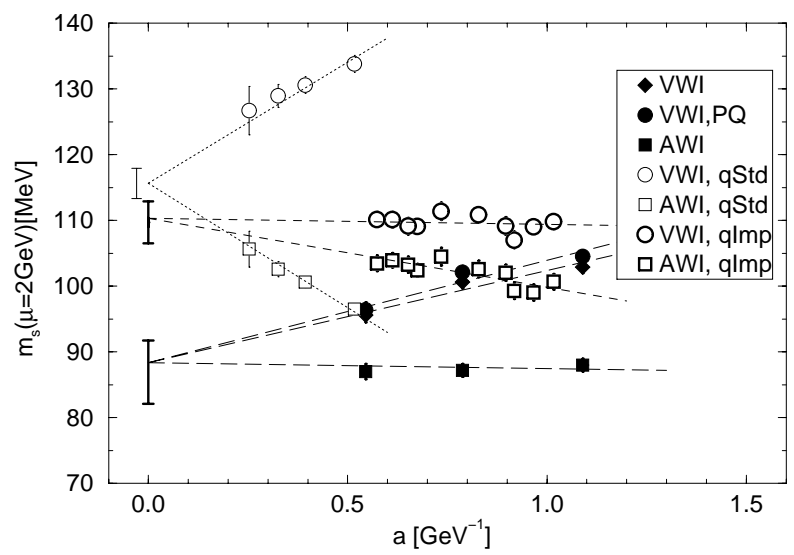

Figure 5. Continuum extrapolations of the strange quark mass computed for $N_{\mathrm{f}}=2$ (solid symbols) and in the quenched approximation (open symbols). A different fermionic discretization used in the quenched case is distinguished by the bold open symbols.

\subsection{Sea quark effects in $m_{\mathrm{s}}$ and $\widehat{m}$}

So far the most comprehensive study of quark masses using simulations with dynamical quarks has been presented recently by CP-PACS [25]. Earlier results can be found in refs. $[33,12]$. When discussing the results, it is important to keep in mind that non-perturbative renormalization has not yet been implemented for $N_{\mathrm{f}}=2$ flavours. Instead the renormalization of the lattice matrix element is usually performed using so-called "mean-field improved" lattice perturbation theory [34]. In addition to computing the strange quark mass, CP-PACS have also estimated the average light quark mass through extrapolations to the chiral limit. Furthermore they have compared different definitions of the quark mass, based either on the axial vector Ward identity (AWI, i.e. the PCAC relation) or the vector Ward identity (VWI).

The continuum extrapolations for the strange quark mass are shown in Fig. 5. Results from the AWI and VWI definitions of the quark mass have been extrapolated enforcing a common continuum limit. By comparing the results for $N_{\mathrm{f}}=2$ to those obtained in the quenched approximation, one finds that the inclusion of dynamical quark effects decreases the estimate for the strange quark mass by roughly $20 \%$. The results for $m_{\mathrm{s}}$ by CP-PACS can be summarized as follows:

$$
m_{\mathrm{s}}^{\overline{\mathrm{MS}}}(2 \mathrm{GeV})=\left\{\begin{aligned}
88_{-6}^{+4} \mathrm{MeV}, & N_{\mathrm{f}}=2 \\
110_{-4}^{+3} \mathrm{MeV}, & \text { quenched } .
\end{aligned}\right.
$$

The light quark mass $\widehat{m}$ shows a similar decrease when sea quark effects are included. However, for both $N_{\mathrm{f}}=2$ and $N_{\mathrm{f}}=0$ (quenched) one finds that $m_{\mathrm{s}} / \widehat{m} \approx 25$ in the continuum limit, which is consistent with the prediction from ChPT, eq. (13).

Figure 5 also illustrates that lattice artefacts are quite substantial, so that an estimation of quark masses at non-zero lattice spacing would be misleading. This underlines once more the importance of the continuum extrapolation. 


\section{Concluding remarks}

Lattice simulations of quenched QCD have reached a level of precision of a few per cent, thanks to increases in computer power and conceptual advances. The picture that has emerged is that the quenched approximation works surprisingly well, as signified by the success in predicting the spectrum of low-lying hadrons. Non-perturbative renormalization and good control over lattice artefacts are the crucial ingredients that lead to precise estimates of the mass of the strange quark. Also, in order to control the region of very light quarks, which cannot be simulated on present machines, it is useful to combine lattice simulations with Chiral Perturbation Theory. In fact, a combination of these techniques may be instrumental in resolving the long-standing question of whether the up-quark is massless [35]. A pilot study along these lines has already appeared in the literature [36].

A major share of computational resources is devoted to studying the effects of dynamical quarks. First results indicate that sea quarks "are doing the right thing", since the discrepancies in the light hadron spectrum between simulation and experiment are decreased. However, more effort is required to understand sea quark effects on a quantitative level. This not only requires larger computers but also the implementation of additional technology such as non-perturbative renormalization for the dynamical case. Finally, there are algorithmic challenges: the efficient simulation of sea quarks for which $m_{\mathrm{PS}} / m_{\mathrm{V}} \ll 0.5$, and also of odd $N_{\mathrm{f}}$ is crucial for the development of lattice QCD into a fully fledged phenomenological tool.

\section{Acknowledgments}

I am grateful to the organizers, in particular Dr Grazyna Odyniec, for the invitation and the offered support. I wish to thank all organizers for creating such a stimulating and pleasant environment at the conference.

\section{References}

[1] Wilson K G 1974 Phys. Rev. D10 2445

[2] Campostrini M et al (ed) 2000 Proc. Int. Symp. on Lattice Field Theory (Lattice 99) Nucl. Phys. B (Proc. Suppl.) 83-84

[3] Kenway R D 2000 plenary talk Int. Conf. on High-Energy Physics (ICHEP 2000) Osaka.

[4] Aoki S 1999 Proc. Int. Symp. on Lepton and Photon Interactions at High Energies (LP 99) Stanford, Preprint hep-ph/9912288

[5] Wittig H 1999, plenary talk Int. Europhysics Conf. on High-Energy Physics (EPS-HEP 99) Tampere, Preprint hep-ph/9911400

[6] Sharpe S 1998 Proc. Int. Conf. on High-Energy Physics (ICHEP 98) Vancouver vol 1 p 171 Preprint hep-lat/9811006

[7] CP-PACS Collaboration (Aoki S et al ) 2000 Phys. Rev. Lett. 84238

[8] Butler F, Chen H, Sexton J, Vaccarino A and Weingarten D 1994 Nucl. Phys. B430 179

[9] MILC Collaboration (Bernard C et al ) 1998 Phys. Rev. Lett. 813087

[10] Kogut J and Susskind L 1975 Phys. Rev. D11 395

[11] UKQCD Collaboration (Bowler K C et al ) 2000 Phys. Rev. D62 054506

[12] SESAM Collaboration (Eicker N et al ) 1999 Phys. Rev. D59 014509

[13] UKQCD Collaboration (Allton C R et al ) 1999 Phys. Rev. D60 034507

[14] UKQCD Collaboration (Garden J et al) 2000 Nucl. Phys. B (Proc. Suppl.) 83-84 165

[15] CP-PACS Collaboration (Ali Khan A et al ) 2000 Nucl. Phys. B (Proc. Suppl.) 83-84 176

[16] Chen $\mathrm{P}$ et al 2000 The finite temperature QCD phase transition with domain wall fermions Preprint hep-lat/0006010.

[17] Particle Data Group (Groom DE et al) 2000 Eur. Phys. J. C3 1

[18] Martinelli G, Pittori C, Sachrajda C T, Testa M and Vladikas A 1995 Nucl. Phys. 44581 
[19] Lüscher M, Narayanan R, Weisz P and Wolff U 1992 Nucl. Phys. B384 168

[20] Sint S 1994 Nucl. Phys. B421 135; 1995 Nucl. Phys. B451 416

[21] Lüscher M 1998 Advanced Lattice QCD Preprint hep-lat/9802029

[22] Capitani S, Lüscher M, Sommer R and Wittig H 1999 Nucl. Phys. B544 669

[23] Leutwyler H 1996 Phys. Lett. B378 313

[24] ALPHA \& UKQCD Collaborations (Garden J et al ) 2000 Nucl. Phys. B571 237

[25] CP-PACS Collaboration (Ali Khan A et al ) 2000 Phys. Rev. Lett. 854674

[26] Becirevic D, Giménez V, Lubicz V and Martinelli G 2000 Phys. Rev. D61 114507

[27] Göckeler M et al 2000 Phys. Rev. D62 054504

[28] Wingate M et al 2000 Nucl. Phys. B (Proc. Suppl.) 83-84 221

[29] Blum T, Soni A and Wingate M 1999 Phys. Rev. D60 114507

[30] JLQCD Collaboration (Aoki S et al) 1999 Phys. Rev. Lett. 824392

[31] Becirevic D et al 1998 Phys. Lett. B444 401

[32] Giménez V, Giusti L, Rapuano F and Talevi M 1999 Nucl. Phys. B540 472

[33] SESAM Collaboration (Eicker N et al ) 1997 Phys. Lett. B407 290

[34] Lepage G P and Mackenzie P B 1993 Phys. Rev. D48 2250

[35] Cohen A G, Kaplan D B and Nelson A E 1999 J. High Energy Phys. JHEP11(1999)027

[36] ALPHA Collaboration (Heitger J, Sommer R and Wittig H) 2000 Nucl. Phys. B588 377 